\title{
Mapeamento de Áreas Suscetíveis a Inundações na Cidade de Duque de Caxias
}

\author{
Douglas Pinto de Oliveiral'; Priscila da Cunha Luz Barcellos ${ }^{2}$; Júlio César da Silva ${ }^{3}$
}

\luz.priscila@gmail.com

1. Pontifícia Universidade Católica do Rio de Janeiro, RJ - Brasil

2. Universidade Federal Fluminense, $R J$ - Brasil

3. Universidade do Estado do Rio de Janeiro, RJ - Brasil.

Histórico do Artigo: 0 autor detém os direitos autorais deste artigo.

Recebido em: 17 de dezembro de 2020 Aceito em: 24 de maio de $2021 \quad$ Publicado em: 31 de dezembro de 2021

\begin{abstract}
Resumo: 0 planejamento urbano e sustentável considerando análises de risco torna-se urgente diante da intensificação de fenômenos naturais extremos. Nos últimos anos, mais de 25.000 pessoas foram afetadas por desastres naturais na cidade de Duque de Caxias (Estado do Rio de Janeiro - Brasil). Esta pesquisa buscou confeccionar e fornecer às autoridades governamentais da cidade de Duque de Caxias uma ferramenta auxiliar com o intuito de direcionar as ações voltadas para prevenção de riscos de inundações, planejamento urbano e desenvolvimento sustentável, através da elaboração do mapa de áreas propensas a inundações. 0 trabalho utilizou o método empírico de combinação da Análise Hierárquica Ponderada (AHP) e a plataforma GIS, dando pesos aos seguintes critérios escolhidos: declividade, altitude e uso e ocupação do solo. Em comparação com os dados de inundações noticiadas entre 1996 e 2015 em Duque de Caxias, os resultados mostraram que, apesar da baixa qualidade de dados para comparação, o mapa conseguiu indicar as áreas mais suscetíveis a inundações, mostrandose ser uma ferramenta útil para gestão de riscos e planejamento urbano na cidade. A pesquisa mostrou que a utilização da AHP para confecção de mapas de risco à inundação pode ser uma metodologia confiável e aplicável a outras cidades do Brasil.

Palavras-chave: Inundações, Mapeamento de risco, Método AHP, Planejamento urbano, Gestão de riscos de desastres.
\end{abstract}

\section{Mapping of Areas Sustainable to Flooding in the Duque de Caxias City}

\begin{abstract}
Urban and sustainable planning considering risk analyzes is urgent given the intensification of extreme natural phenomena. In recent years, more than 25,000 people have been affected by natural hazards in the city of Duque de Caxias (State of Rio de Janeiro - Brazil). This research sought to prepare and provide governmental authorities in the city of Duque de Caxias with an auxiliary tool in order to direct actions aimed at preventing flood risks, urban planning and sustainable development, through the elaboration of the map of areas prone to flooding. The work used the empirical method of combining Analytic Hierarchy Process (AHP) and the GIS platform, giving weights to the following chosen criteria: slope, altitude and land use and occupation. In comparison with the flood data reported between 1996 and 2015 in Duque de Caxias, the results showed that, despite the low quality of data for comparison, the map managed to indicate the areas most susceptible to flooding, proving to be a useful tool for risk management and urban planning in the city. The research showed that the use of AHP for making flood risk maps can be a reliable methodology and applicable to other cities in Brazil.
\end{abstract}

Keywords: Flood, Risk mapping, AHP method, Urban planning, Disaster risk management. 


\section{Cartografía de Zonas Sostenibles a Inundaciones en la Ciudad de Duque de Caxias}

Resumen: La planificación urbana y sostenible considerando análisis de riesgo es urgente dada la intensificación de fenómenos naturales extremos. En los últimos años, más de 25.000 personas se han visto afectadas por desastres naturales en la ciudad de Duque de Caxias (Estado de Río de Janeiro - Brasil). Esta investigación buscó preparar y dotar a las autoridades gubernamentales de la ciudad de Duque de Caxias de una herramienta auxiliar para dirigir acciones encaminadas a la prevención de riesgos de inundaciones, planificación urbana y desarrollo sostenible, mediante la elaboración del mapa de áreas propensas a inundaciones. El trabajo utilizó el método empírico de combinar el análisis jerárquico ponderado (AHP) y la plataforma GIS, dando ponderaciones a los siguientes criterios elegidos: pendiente, altitud y uso y ocupación del suelo. En comparación con los datos de inundaciones reportados entre 1996 y 2015 en Duque de Caxias, los resultados mostraron que, a pesar de la baja calidad de los datos de comparación, el mapa logró señalar las áreas más susceptibles a inundaciones, demostrando ser una herramienta útil para gestión de riesgos y urbanismo en la ciudad. La investigación mostró que el uso de AHP para elaborar mapas de riesgo de inundaciones puede ser una metodología confiable y aplicable a otras ciudades de Brasil.

Palabras clave: Inundaciones, Mapeo de riesgos, Método AHP, Planificación urbana, Gestión del riesgo de desastres.

\section{INTRODUÇ̃̃o}

A ocorrência de fenômenos naturais extremos, como fortes chuvas e inundações, não é considerado como um desastre natural se não produzir impacto em uma população. 0 impacto de um desastre natural depende não somente das características, probabilidade e intensidade do fenômeno natural, assim como da vulnerabilidade das condições geofísicas, socioeconômicas e ambientais das regiões expostas (KOBIYAMA et al., 2006; BOUWER, 2011; CHANG; CHEN, 2016).

No Brasil, 59\% dos registros dos desastres naturais são ocasionados por inundações. Com exceção das inundações graduais, as rápidas inundações, denominadas enxurradas segundo a Codificação Brasileira de Desastres - COBRADE (RODRIGUES et al., 2015), são súbitas e violentas, sendo responsáveis por grandes perdas humanas e materiais (DAHRI; ABIDA, 2017; KAUR et al., 2017). Em virtude de sua velocidade, o tempo necessário para que a população, que reside nas áreas de risco, busque abrigos ou salvem parte de seus bens materiais é extremamente limitado (MARCELINO, 2007).

Diante disso, duas áreas de gestão de risco estão interligadas, visando a mitigação e prevenção aos desastres naturais: as ciências ambientais e sociais. Atualmente, cresce a preocupação com ambas as áreas, pois, se por um lado pesquisadores apontam que possíveis mudanças climáticas indicam aumento na severidade das precipitações extremas para os próximos anos (TEBALDI et al., 2007; MARENG0 et al., 2009; IPCC, 2012); por outro lado, o acelerado processo de urbanização também apresenta preocupações, pois mais da metade da população mundial (50,9\%) já está concentrada nas grandes cidades (R0DRIGUES et al., 2015), o 
que aumenta o risco potencial de ocorrência de desastres, especialmente nas regiões com maior densidade populacional.

No Brasil, o aumento da ocorrência de inundações, assim como de outros desastres naturais, está intimamente relacionado com o aumento da vulnerabilidade da população e ao processo de urbanização, que contribui para o crescimento das cidades em áreas geomorfológicas impróprias para a ocupação. Normalmente, o crescimento urbano nessas áreas é acompanhado por intervenções antropogênicas, como desmatamentos, cortes de taludes, aterros e mudanças da drenagem natural do local, e, na maioria das vezes, não possui infraestrutura adequada de saneamento básico e coleta de lixo, tornando as regiões mais vulneráveis ainda aos desastres naturais (TOMINAGA; SANTOR0; AMARAL, 2009).

No estado de São Paulo, o estudo precursor de geomorfologia de Ab’Saber (1957), mostrou que, por séculos, os assentamentos urbanos ficaram restritos a locais de áreas mais altas e terrenos menos sujeitos a inundações, e que as planícies de inundação natural dos cursos hídricos eram consideradas como elementos impeditivos à expansão urbana no entorno do rio Pinheiros, por exemplo. Porém, a urbanização das cidades atuais se deu com a ocupação urbana dentro das planícies de inundação, o que favorece a problemas como o das enchentes e inundações.

Nas áreas urbanizadas, as decisões políticas e de investimento precisam ser capazes de ajustar o papel dos recursos financeiros no contexto da dinâmica urbana (DEAL; PALLATCHUCHERIL, 2009). Por isso, investir em redução risco, é, acima de tudo, investir no desenvolvimento de uma cidade e uma condição prévia para o desenvolvimento sustentável, pois a erradicação de fenômenos meteorológicos extremos, que são os precursores dos principais desastres naturais no Brasil, é praticamente impossível (MARCELINO, 2007).

Fazendo parte do histórico dos estudos de geomorfologia derivados do trabalho de Ab’Saber (1957) como ferramentas de combate a catástrofes urbanas, para o planejamento urbano pode-se citar como exemplo as cartas geotécnicas de ocupação de morros, um documento cartográfico contendo informações sobre as características do meio físico e seus problemas existentes ou esperados, como as utilizadas em Santos (SP), e o Plano de Erradicação de 0cupação de Encostas, da cidade de Belo Horizonte - MG.

Dessa forma, a gestão de risco deve ser direcionada com o objetivo de implementar medidas mitigadoras e preventivas que possam diminuir o impacto ocasionado pelos desastres naturais (YOUSSEF; PRADHAN; SEFRY, 2016; KLAUSS et al., 2016). No caso das inundações, medidas estruturais, como por exemplo obras de contenção de encostas, e não estruturais, como por 
exemplo aquelas por meio de políticas e leis, conscientização pública, treinamento e educação, são aplicadas em todo o mundo visando a prevenção dos desastres naturais (NCAR, 2010; SUN; ZHANG; CHENG, 2012; LUZ BARCELLOS et al., 2017), sendo os métodos não estruturais mais utilizados, principalmente, porque os estruturais são financeiramente muito caros e não fornecem 100\% de proteção contra inundações (FAISAL et al., 1999).

Entre os métodos não estruturais, o mapa de risco de inundações com alta resolução espacial é essencial para o gerenciamento de recursos hídricos e ambientais, para resposta a emergências, proteção de vidas e propriedades (FEMA, 2003; WM0, 2009; ZÊZERE et al., 2014; BATHRELLOS et al., 2016; SHAHABI; HASHIM; AHMAD, 2015; AL-ABADI; SHAHID; AL-ALI, 2016; MIRZAEI et al., 2018).

Para a confecção dos mapas de risco de inundações, técnicas de mapeamento que utilizam o método do Análise Hierárquica Ponderada (AHP) (SAATY, 1977) ganham destaque como uma aproximação indireta e complementar a estudos em diversas áreas ambientais. Trabalhos sobre mapeamento de inundações e alagamentos, como os realizados por Coelho (2017) para 0 município de Vitória (ES), Miranda et al. (2019) em Cuiabá (MT), Nogueira (2017) para o município de Patos (MG), Campioli e Vieira (2019) para Joinville (SC), Camargo Júnior et al. (2018) para a Bacia Ribeirão Anhumas em Campinas (SP), Braga et al. (2020) para a cidade de Limoeiro (PE), e para mapeamento de escorregamento de encostas feito por Faria e Augusto Filho (2013) para a cidade de São Sebastião (SP) e Silva e Riedel (2018) para o estado do Rio de Janeiro, são realizados como uma forma de auxiliar a gestão de recursos hídricos, desastres naturais e as ações governamentais junto as comunidades em risco.

No levantamento feito com relatos de mídia, Luz Barcellos et al. (2017) mostrou que na cidade de Duque de Caxias, localizada na Baixada Fluminense do estado do Rio de Janeiro e importante por possuir uma das maiores refinarias de Petróleo do Brasil, somente entre 1996 a 2015 sofreu com 35 desastres naturais, deixando mais de 25.000 pessoas afetadas, e mais de 70 mortes relacionadas a fortes chuvas, inundações e deslizamentos de encostas. Em janeiro de 2013, uma forte enxurrada assolou vários bairros, destruindo ruas e casas, e devastando partes importantes da cidade (LUZ BARCELLOS, 2016). Após esse desastre, diversas ações governamentais foram iniciadas na cidade com o objetivo de modernizar a defesa civil, por meio de decretos, normas e acordos nacionais e internacionais (LUZ BARCELLOS et al., 2017).

Nesse contexto, esta pesquisa tem como objetivo elaborar o mapa de áreas propensas à inundação para a cidade de Duque de Caxias, utilizando o método AHP, através da combinação das variáveis geomorfológicas de altitude, declividade e uso e ocupação do solo, utilizando imagens de satélite do banco de dados do Shuttle Radar Topography Mission (SRTM) e 
informações de uso e ocupação do solo da cidade disponibilizadas pelo Sistema de Informação Geográfico da Região Hidrográfica da Baia de Guanabara (SIG-RHBG), do Comitê de Bacia da Baia de Guanabara.

0 mapeamento feito através de uma metodologia determinística, simples e de fácil aplicação, busca elaborar uma ferramenta que dê a diretriz inicial para os órgãos governamentais, auxiliando não somente na tomada de decisão em caso de ocorrência de desastres naturais do tipo inundações e enxurradas, mas também no gerenciamento ambiental e no desenvolvimento sustentável da cidade.

\section{MATERIAL E MÉTODOS}

\section{Região de Estudo}

0 município de Duque de Caxias (RJ) localiza-se na região da Baixada Fluminense e é caracterizado por uma extensão territorial de $467.319 \mathrm{~km}^{2}$, e possui 924.624 habitantes (IBGE, 2020). Os limites espaciais se estendem desde Baía de Guanabara, ao sul, até o início da Serra do Mar, ao norte, e a cidade apresenta características de baixada, com áreas de planície baixa e alto risco às inundações em períodos chuvosos, e, ainda, está sob a influência das marés, formada por rios que drenam para a Baía de Guanabara (IORIS; COSTA, 2009), apresentando algumas regiões com altitude negativa (MONTEZUMA; OLIVEIRA, 2010).

Segundo o IBGE (2015), a cidade de Duque de Caxias ocupa o equivalente a 6,8\% da Região Metropolitana do Estado do Rio de Janeiro e, aproximadamente, 35\% da região chamada Baixada Fluminense, com cerca de 99\% da população residente nas áreas urbanas.

Duque de Caxias possui um parque industrial significativo, abrigando além da $2^{\mathrm{a}}$ maior refinaria do país, a Refinaria de Duque de Caxias (REDUC), outros segmentos industriais como o metalúrgico, mobiliário e têxtil. Administrativamente, está dividido em quatro Distritos, com a população caxiense concentrada no primeiro distrito do município, com a maior densidade demográfica da região devido principalmente a maior proximidade com a cidade do Rio de Janeiro.

0 processo de industrialização na década de 1960, com a instalação da REDUC no $2^{\circ}$ Distrito, influenciou a migração da população para essa região diante da oferta de empregos, o que levou a população mais humilde a ocupar terrenos inundáveis ao longo dos cursos hídricos (PREVOT et al., 2014). A longo prazo, estas ocupações geraram níveis graves de poluição e degradação dos rios, e, também a remoção da mata ciliar, resíduos não recolhidos e a 
impermeabilização de solos, agravando os problemas relacionados com as inundações (IORIS; COSTA, 2009).

Com o clima tropical e precipitação média mensal entre 300 e 400 mm durante os meses de verão (INMET, 2020), a ocorrência de fenômenos atmosféricos extremos aliado ao crescimento desordenado da cidade, juntamente com a topografia e a hidrografia do município, evidenciando cenários de vulnerabilidade da população, tornam 0 município ainda mais favorável à ocorrência de desastres naturais.

Como não há um histórico de dados de ocorrências de desastres naturais na cidade, Luz Barcellos et al. (2017) identificaram 35 grandes eventos entre os anos de 1996 e 2015, através de um levantamento na mídia. A pesquisa considerou como desastres os eventos que resultaram em pelo menos um dos seguintes aspectos: ocorrência de desabrigados e/ou desalojados, mortes, deslizamentos de terra ou inundações de rios (mesmo sem vítimas), declaração de situação de emergência ou declaração de estado de calamidade pública.

Mesmo com o levantamento das datas dos desastres ocorridos obtidos a partir da pesquisa de Luz Barcellos et al. (2017), a identificação do local exato e dos bairros afetados na mídia onde as inundações ocorreram não estavam disponíveis. A figura 1 apresenta o número de inundações ocorridas por bairros e descritas por Luz Barcellos et al. (2017), onde, dos 35 eventos citados no estudo, apenas 19 deles tinham informações do bairro afetado. 


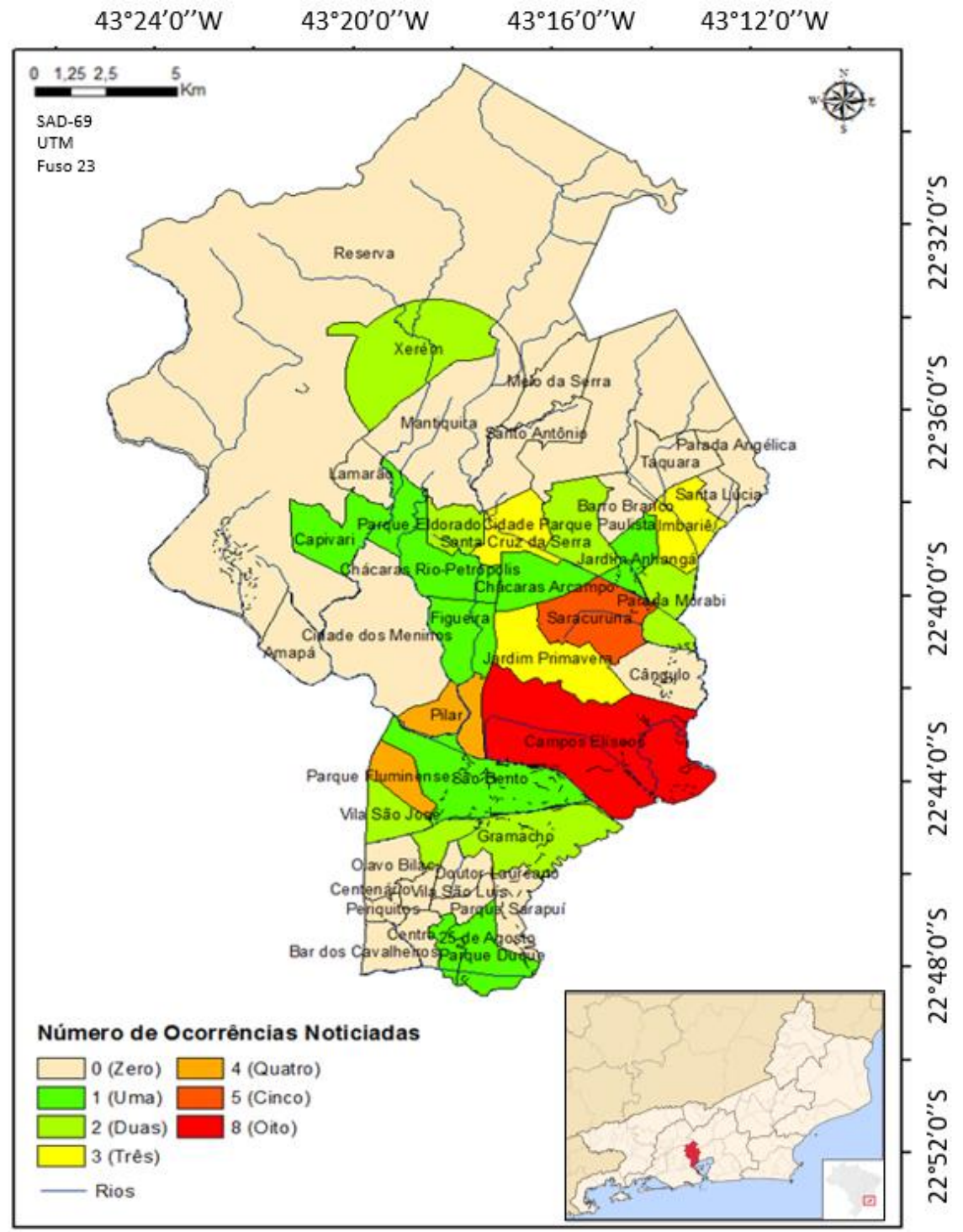

Figura 1. Número de ocorrência de inundações por bairro na cidade de Duque de Caxias entre 1996 e 2015.

Fonte: Adaptado e modificado de Luz Barcellos et al. (2017) e Wikipédia.

Apesar da baixa qualidade dos dados, na figura 1 é possível destacar que os bairros localizados na região centro-sul da cidade, mais precisamente no $2^{\circ}$ Distrito, como nos bairros de Campos Elíseos, Jardim Primavera e Pilar, foram os mais atingidos por inundações nos registros noticiados.

\section{Metodologia}

Para a construção do mapa de áreas propensas a inundações, esta pesquisa utilizou 0 método AHP, que consiste num método de instrumento racional de decisão de um problema em níveis hierárquicos (SAATY, 1977), classificando os fatores envolvidos em uma hierarquia de critérios com atribuição de pesos para cada um. 
Uma vez que os critérios de comparação e os pesos de importância relativos entre os critérios são estabelecidos, calcula-se a razão de consistência (RC) dos julgamentos, o que permite verificar se a atribuição de pesos relativos a cada par de critérios estabelecidos possui valores aceitáveis (SAATY, 1980; FARIA; AUGUST0 FILH0, 2013). Só assim foi feito um modelo matemático que calcula o risco de inundação para a cidade de Duque de Caxias.

Para análise dos critérios estabelecidos e para a confecção do mapa foram utilizados: 0 software ArcMap 10.2.2., da plataforma ArcGIS, as imagens de satélite do Shuttle Radar Topography Mission (SRTM) com resolução espacial de 30 metros para as análises dos critérios de altitude e de declividade do terreno, e as informações de uso e ocupação do solo da cidade de Duque de Caxias disponibilizadas pelo Sistema de Informação Geográfico da Região Hidrográfica da Baia de Guanabara (SIG-RHBG), do Comitê de Bacia da Baia de Guanabara, com resolução espacial de 20 m para as análises de urbanização do terreno.

0 método AHP baseia-se em três fases: 1. Atribuição de pesos, 2. Cálculo da matriz de comparação do vetor de prioridades, e 3. Análise da consistência dos julgamentos, apresentados a seguir.

\section{Atribuição de pesos e escolha de critérios}

Destacado como um dos processos mais importantes na confecção do mapa (SANTOS et al., 2010), os critérios escolhidos foram comparados par a par com base na escala de julgamentos, denominada Escala Fundamental de Saaty (1980), para a definição do grau de importância de cada critério na ocorrência da inundação, onde o valor 1 equivale ao mínimo, e 9 ao máximo de importância de um fator sobre o outro.

Nesta pesquisa, foi utilizado a escala somente com valores ímpares, usados normalmente para assegurar uma distinção razoável entre os pontos de medição (SAATY, 1980), pois os valores pares somente são adotados se não houver consenso comum entre os avaliadores dos pesos das variáveis (FARIA; AUGUSTO FILH0, 2013).

Na tabela 1 é apresentada a escala comparativa utilizada nesta pesquisa, onde os valores maiores que 1 significam que a variável possui uma importância relativamente maior do que a outra, e os valores abaixo de 1 mostram uma comparação inversa, onde os valores indicam que a variável é menos importante que a variável em comparação.

Tabela 1. Escala de julgamento de importância no Método AHP.

\begin{tabular}{cc}
\hline VALORES & IMPORTÂNCIA MÚTUA \\
\hline $1 / 9$ & Extremamente menos importante que
\end{tabular}




$\begin{array}{cl}1 / 7 & \text { Muito fortemente menos importante que } \\ 1 / 5 & \text { Fortemente menos importante que } \\ 1 / 3 & \text { Moderadamente menos importante que } \\ 1 & \text { Igualmente importante a } \\ 3 & \text { Moderadamente mais importante que } \\ 5 & \text { Fortemente mais importante que } \\ 7 & \text { Muito fortemente menos importante que } \\ 9 & \text { Extremamente mais importante que }\end{array}$

Fonte: Modificado de Saaty (1990).

Os critérios escolhidos para a elaboração do mapa levaram em consideração aqueles que poderiam influenciar mais significativamente no processo de formação das inundações na cidade de Duque de Caxias, e que também foram destacados na revisão de literatura de Leal et al. (2020) como as variáveis principais que impactam na inundação de rios e utilizadas em todos os trabalhos analisados pelos autores: altitude, declividade e uso e cobertura do solo.

Analisando a cidade, observa-se que o município é composto por grandes variações de altitude, com alguns bairros atingindo valores de altitude abaixo do nível médio do mar, sendo, desta forma, considerado o critério mais importante nesta pesquisa. Segundo Magalhães et al. (2011), a altitude é caracterizada como um critério muito importante, pois quanto maior for esse critério, menor a probabilidade de ocorrência de inundações devido à ação da lei da gravidade na superfície, que direciona a água das regiões mais altas para as mais baixas.

0 critério de declividade do terreno foi considerado o segundo mais importante no estudo devido ao fato da cidade ser formada principalmente por áreas planas, com declives abaixo de 8\%, o que influencia diretamente o acúmulo de água no terreno, pois áreas planas são mais favoráveis à ocorrência de inundações do que áreas íngremes.

E, como terceiro critério considerado, e não menos importante, o uso e cobertura do solo tem sua importância relacionada à infiltração e escoamento da água, onde áreas com maior impermeabilidade tendem a acumular mais água na superfície do que os solos com cobertura florestal, por não serem muito compactados. A cidade de Duque de Caxias é caracterizada por muitos trechos com cobertura impermeável do terreno, com cerca de 99\% da população residente nas áreas urbanas.

Com os critérios geomorfológicas pré-definidos: a altitude, a declividade e 0 uso e cobertura do solo, esses foram classificados de acordo com o grau de suscetibilidade a inundações, com pesos variando de 0 a 10 , onde 0 corresponde à menor suscetibilidade a inundações e 10 a maior suscetibilidade. 


\section{Cálculo da matriz de comparação do vetor de prioridades}

A partir da tabela 1, foi construída a matriz de comparação de cada critério em relação ao outro critério, estruturada de maneira que o critério 1 tenha preferência sobre o critério 2. Nesta pesquisa, os critérios seguiram a seguinte ordem de importância: altitude, declividade e uso e cobertura do solo.

Assim, foram atribuídos graus de importância aos critérios através da comparação pareada e recíproca (tabela 2). Cada elemento da matriz indica o quanto cada critério da coluna da esquerda é mais importante em relação à suscetibilidade à inundação avaliado com cada critério correspondente na linha superior. 0 somatório dos critérios de cada coluna corresponde aos pesos de cada elemento relativos a eles mesmos, e serão utilizados para a normatização dos critérios.

Tabela 2. Matriz de comparação pareada e soma dos critérios de cada coluna.

\begin{tabular}{cccc}
\hline CRITÉRIOS & $\begin{array}{c}\text { Uso e cobertura } \\
\text { do solo }\end{array}$ & Declividade & Altitude \\
\hline Uso e cobertura do solo & 1 & $1 / 3$ & $1 / 5$ \\
Declividade & 3 & 1 & $1 / 3$ \\
Altitude & 5 & 3 & 1 \\
Total & 9 & 4,3333 & 1,5333 \\
\hline
\end{tabular}

Fonte: 0 autor.

0 cálculo da matriz de comparação resulta no chamado "autovetor de prioridades" (FARIA; AUGUSTO FILHO, 2013), e dividindo-se a soma de cada linha dos critérios pela soma dos elementos da matriz, determina-se o peso relativo de cada critério (SAATY, 1990).

A tabela 3 apresenta a normalização relativa desses pesos calculados através da divisão de cada elemento da coluna pela somatória dos elementos da coluna a que ele pertence, respectivamente. Na coluna da direita observa-se a normalização do autovetor ou peso final, que após encontrar os pesos específicos para cada variável, o autovetor é calculado através da média aritmética de cada linha da matriz.

Tabela 3. Cálculo dos pesos para cada variável e normalização do autovetor.

\begin{tabular}{cccccc}
\hline Critérios & $\begin{array}{c}\text { Uso e } \\
\text { cobertura do } \\
\text { solo }\end{array}$ & Declividade & Altitude & $\begin{array}{c}\text { Peso } \\
\text { final }\end{array}$ & $\begin{array}{c}\text { Autovetor } \\
\text { (\%) }\end{array}$ \\
\hline $\begin{array}{c}\text { Uso e cobertura } \\
\text { do solo }\end{array}$ & $1 / 9=0,1111$ & $0,3333 / 4,3333=$ & $0,2 / 1,5333=$ & 0,1061 & 10,61 \\
Declividade & $3 / 9=0,3333$ & $1 / 4,3333=0,2308$ & $0,3333 / 1,5333=$ & 0,2605 & 26,05
\end{tabular}




$\begin{array}{llllll}\text { Altitude } & 5 / 9=0,5556 & 3 / 4,3333=0,6923 & 1 / 1,5333=0,6522 & 0,6334 & 63,34 \\ \text { Fonte: } 0 \text { autor. }\end{array}$

\section{Análise da consistência dos julgamentos}

A análise da consistência dos julgamentos refere-se a Razão de Consistência (RC) das avaliações, dada pela fórmula $\mathrm{RC}=\mathrm{IC} / \mathrm{IR}$, onde IC é o Índice de Consistência e IR é o Índice Randômico, que indica o valor da matriz de comparação paritária que seria encontrada sem realizar os julgamentos (FARIA; AUGUST0 FILH0, 2013). Segundo Saaty (2008), o resultado da consistência dos julgamentos deve obter valores de $\mathrm{RC} \leq 0,10$, pois valores acima significam que há inconsistências de julgamentos e 0 uso do método AHP não será adequado.

Para calcular o autovalor, foi calculada a matriz $A w$ multiplicando a matriz de comparação pareada pelos respectivos pesos calculados na tabela 3.

\section{Confecção do mapa de áreas propensas a inundação}

Confirmado a veracidade dos pesos, um modelo matemático foi construído para descrever a suscetibilidade à ocorrência de inundações na cidade, onde:

$$
S=\left(P_{1} \cdot C_{1}\right)+\left(P_{2} \cdot C_{2}\right)+\left(P_{3} \cdot C_{3}\right)
$$

sendo $S$ a suscetibilidade, $P_{1}, P_{2}$ e $P_{3}$ os pesos relativos e $C_{1}, C_{2}$ e $C_{3}$ os critérios estabelecidos para 0 cálculo.

Os resultados de $S$ foram classificados em cinco faixas de suscetibilidade à inundação:

- Baixo-baixíssimo e médio-baixo: áreas urbanas com baixa ou média probabilidade de ocorrências de inundações, esperados baixos ou médios danos e prejuízos;

- Médio: áreas urbanas com média probabilidade de ocorrências de inundações, esperados médios danos e prejuízos;

- Médio-alto e alto-altíssimo: áreas urbanas com alta probabilidade de ocorrências de inundações, esperados altos danos e prejuízos.

\section{RESULTADOS E DISCUSSÃ0}

Os critérios de altitude, declividade e uso e cobertura do solo foram subdivididos em classes de suscetibilidade à inundação, aos quais foram atribuídos pesos que variaram entre 1 e 10 de acordo com o grau de importância na suscetibilidade à inundação, sendo pesos próximos 
a 1 para classes com menor influência na suscetibilidade, e aumentando progressivamente até 10 segundo sua influência (CAPRARI0 et al., 2019).

\section{Critério 1. Altitude}

Os pesos de classificação, apresentados na tabela 4, considera altitudes mais baixas com pesos mais altos, indicando maior suscetibilidade à inundação e, maiores altitudes com pesos mais baixos.

Tabela 4. Pesos estabelecidos para o critério altitude segundo o grau de suscetibilidade a inundações.

\begin{tabular}{cc}
\hline ALTITUDE $(\mathrm{m})$ & PESOS \\
\hline$\leq 2$ & 10 \\
$2-4$ & 9 \\
$4-6$ & 8 \\
$6-8$ & 7 \\
$8-10$ & 6 \\
$10-50$ & 5 \\
$50-100$ & 4 \\
$100-500$ & 3 \\
$500-1000$ & 2 \\
$1000-1500$ & 1 \\
\hline
\end{tabular}

\section{Critério 2. Declividade}

As faixas de declividade e seus respectivos pesos para sua classificação são apresentadas na tabela 5, onde as áreas mais planas recebem pesos maiores, e áreas mais ondulosas e/ou montanhosas recebem pesos menores, variando de acordo com o aumento das características de ondulação do solo nas proximidades das montanhas.

Tabela 5. Pesos estabelecidos para o critério declividade segundo o nível de suscetibilidade à inundação.

\begin{tabular}{lc}
\hline Declividade (\%) & Peso \\
\hline $0-3$ (plano) & 10 \\
$3-8$ (suavemente ondulado) & 9 \\
$8-20$ (ondulado) & 5 \\
$20-45$ (fortemente ondulado) & 3 \\
$45-75$ (montanhoso) & 1 \\
$75-100$ (fortemente montanhoso) & 1
\end{tabular}

Fonte: Borges et al. (2015). 


\section{Critério 3. Uso e cobertura do solo}

A tabela 6 apresenta os tipos de uso e cobertura de solo e seus respectivos pesos atribuídos a suscetibilidade a inundações, onde áreas de solo exposto, florestas ou baixa ocupação territorial receberam pesos menores, e áreas com alta densidade populacional, pastagens, mangues e áreas úmidas receberam pesos maiores.

Tabela 6. Pesos estabelecidos para o critério uso e cobertura do solo segundo o nível de suscetibilidade à inundação.

\begin{tabular}{lc}
\hline Uso e cobertura do solo & Peso \\
\hline Águas & 10 \\
Ocupação urbana de alta densidade & 9 \\
Pastagem & 8 \\
Pastagem de várzea & 8 \\
Agricultura & 8 \\
Mangue & 8 \\
Áreas úmidas & 8 \\
Ocupação urbana de média densidade & 7 \\
Solo exposto & 7 \\
Ocupação urbana de baixa densidade & 5 \\
Floresta & 1 \\
Vegetação secundária em estágio inicial & 1 \\
Afloramento rochoso & 1 \\
\hline galhães et al. (2011), Dalfi et al. (2013), Silveira et al. (2013) e Borges et al. (2015).
\end{tabular}

\section{Método AHP aplicado na análise da susceptibilidade à inundação}

0 resultado do autovetor (tabela 3 ) indica que 0 critério da altitude tem importância de $63,34 \%$, a declividade de $26,05 \%$ e o uso e cobertura do solo com importância de $10,61 \%$ em relação ao risco de inundação na cidade de Duque de Caxias.

Através do cálculo da matriz $A w$, foi obtido o valor de 3,0066 do autovetor. Desta forma, pode-se calcular o índice de consistência (IC), onde:

$$
I C=\frac{3,0066-3}{2}=0,0033
$$

onde $I C$ é o índice de consistência, 3,0066 o valor do autovetor e 3 o valor de $n$ que é a dimensão da matriz utilizada.

Retirando o valor do IR de Saaty (2008), para $n=3$ o IR possui 0 valor de 0,58. Assim, calculou-se a razão de consistência (RC), sendo 


$$
R C=\frac{0,0033}{0,58}=0,0057
$$

0 valor obtido de 0,0057 para o $\mathrm{RC}$ indica que há consistência de julgamentos e 0 resultado do uso do método AHP é considerado satisfatório, validando os critérios escolhidos e os pesos relativos dado a cada elemento da matriz.

\section{Mapa de suscetibilidade à inundação na cidade de Duque de Caxias}

A elaboração do mapa de suscetibilidade ao risco de inundação foi confeccionada utilizando a equação 1, onde

$S=(0,1061 x$ uso e cobertura do solo $)+(0,2605 x$ declividade $)+$ $(0,6334 x$ altitude $)$

sendo $S$ a suscetibilidade, $P_{1}=0,1061, P_{2}=0,2605$ e $P_{3}=0,6334$ os pesos relativos a cada critério.

0 resultado do mapa de suscetibilidade a inundações (figura 2), apresenta as regiões localizadas no centro-sul da cidade com alta suscetibilidade a inundações, mostrando que os critérios de altitude e declividade foram determinantes para o grau de perigo a ocorrência do fenômeno nessas regiões.

Nas regiões localizadas próximas a Serra do Mar, ao norte do município, a suscetibilidade a inundações médio-baixo à baixo-baixíssimo evidencia a importância dos pesos dados aos critérios escolhidos, pois além da região possuir altitude elevada e alta declividade, também é uma área com baixa densidade populacional. 


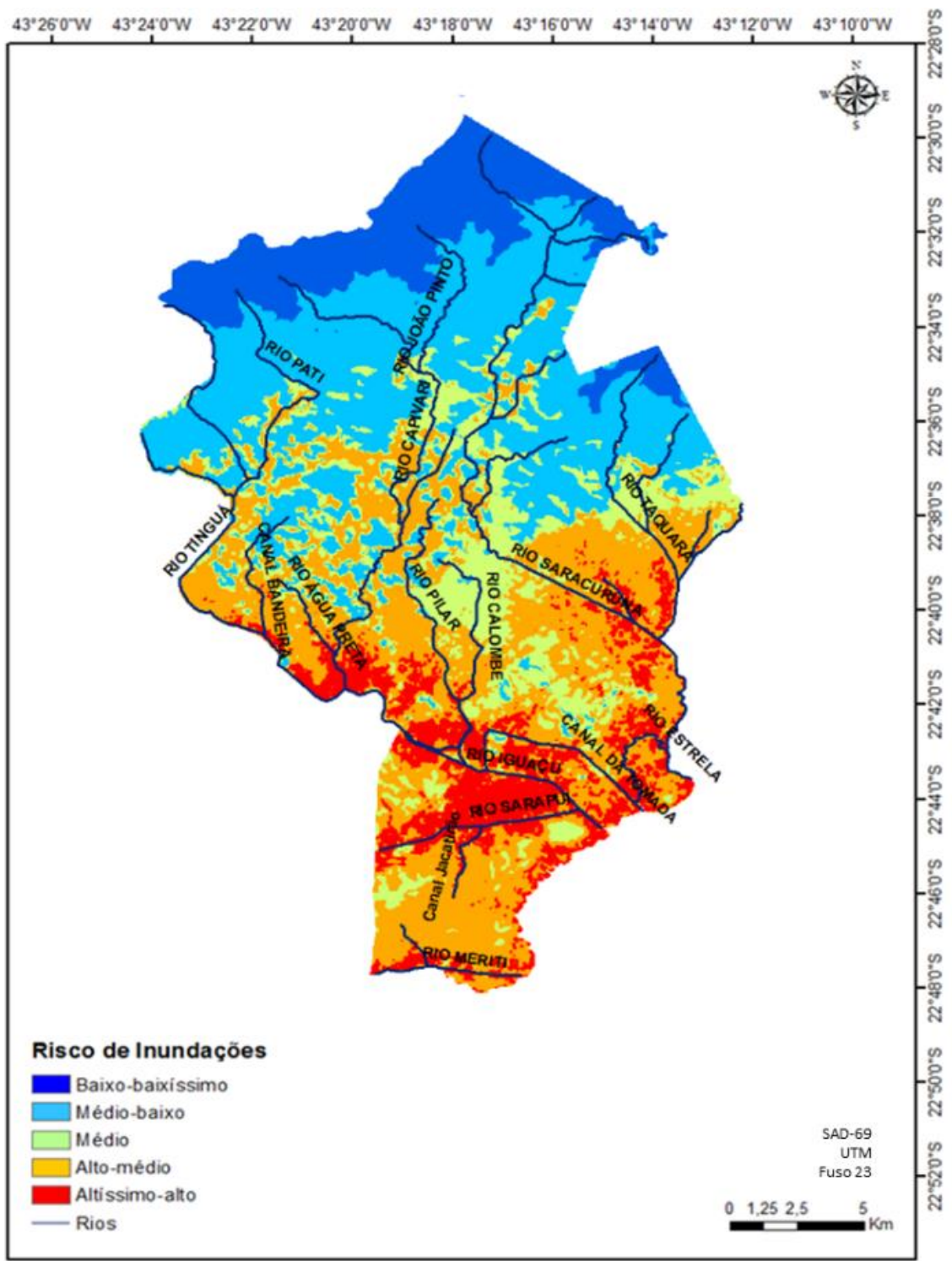

Figura 2. Mapa de áreas propícias a inundações para a cidade de Duque de Caxias. Fonte: 0 autor.

Em resumo, a ponderação integrada dessas variáveis caracterizou a porção centro-sul da cidade de Duque de Caxias como a mais vulnerável à ocorrência de inundações, diminuindo progressivamente quando em direção à porção norte do município.

Em comparação visual com as inundações noticiadas (figura 1), é possível destacar que os bairros no $2^{0}$ Distrito, como Campos Elíseos, Jardim Primavera e Pilar, classificados como risco altíssimo-alto, foram os mais atingidos por inundações nos registros noticiados. Já o bairro de São Bento, classificado em grande parte como suscetibilidade alta, apresentou apenas um registro.

No extremo norte da cidade, com exceção do bairro de Xerém que apresenta suscetibilidade ao risco alto-médio devido principalmente a sua ocupação territorial, as demais 
regiões foram classificadas com suscetibilidade baixa, o que corrobora com o não registro da mídia de inundações ocorridas entre 1996 e 2015.

Apesar da baixa qualidade dos dados históricos e da comparação visual aproximada, os critérios e os pesos utilizados para confecção do mapa mostraram que a metodologia é viável como uma primeira ferramenta para mapeamento em relação a possibilidade de inundações na cidade de Duque de Caxias.

\section{CONCLUSÕES}

Nesta pesquisa, foi utilizada uma abordagem empírica através da integração dos métodos AHP e GIS para a produção do mapa de suscetibilidade a inundações, com o objetivo de auxiliar na gestão ambiental e de risco, na prevenção de desastres naturais na cidade de Duque de Caxias.

Elencando os critérios da altitude, declividade e uso e ocupação do solo, a metodologia proposta considerou informações sobre variáveis geomorfológicas, dando peso relativo à importância de cada um deles no processo de inundação, a fim de identificar os pontos críticos e regiões mais favoráveis aos desastres naturais do tipo de inundação na cidade. Um modelo matemático foi proposto e, através do cálculo para a região analisada, foi possível a construção do mapa de suscetibilidades.

Os resultados mostraram, de forma geral e em macro escala, grande similaridade entre o mapa de suscetibilidade a inundações e a ocorrência de inundações por bairros noticiados pela imprensa entre 1996 e 2015. A porção centro-sul da cidade de Duque de Caxias, destacada como aquela que apresentou maior suscetibilidade a inundações, foi também a região onde foram noticiados o maior número de bairros com ocorrência de inundação. Também foi possível observar uma diminuição gradativa da suscetibilidade em direção a porção norte do município de Duque de Caxias, regiões onde não foram noticiados registros de inundação no período estudado.

A pesquisa demonstrou que metodologia de integração método AHP-GIS pode ser uma abordagem fácil e confiável para a confecção de mapas de suscetibilidade a inundações, auxiliando para a prevenção e mitigação de desastres naturais e no gerenciamento ambiental, e ressalta a importância da ferramenta acadêmica como guia inicial para políticas públicas.

Como pesquisas futuras, sugere-se que o método de integração AHP-GIS seja calculado com outros dados e com a mesma metodologia, avaliando as diferenças que ocorrerão no mapeamento, assim como seja aplicado e analisado para outros municípios do estado do Rio de 
Janeiro, permitindo uma visão estadual do fenômeno, e, ainda, seja refinado para bairros e ruas. Sugere-se também que o mapeamento seja um instrumento constituinte e inicial para a construção de um plano diretor ambiental urbano, que busque o controle de expansão em áreas urbanas ambientalmente suscetíveis a grandes impactos ambientais.

\section{REFERÊNCIAS BIBLIOGRÁFICAS}

AB’SABER, A.N. Geomorfologia do sítio urbano de São Paulo. Cotia, SP: Ateliê, 2007 [1957].

AL-ABADI, A.M., SHAHID, S., AL-ALI, A.K. A GIS-based integration of catastrophe theory and analytical hierarchy process for mapping flood susceptibility: a case study of Teeb area, Southern Iraq. Environmental Earth Sciences, v. 75 , p. 687,2016 .

BOUWER, L.M. Have disaster losses increased due to anthropogenic climate change? Bulletin of the American Meteorological Society, v. 92, n. 1, p. 39-46, 2011.

BATHRELLOS, G.D., KARYMBALIS, E., SKILODIMOU, H.D., GAKI-PAPANASTASSIOU, K., BALTAS, E.A. Urban flood hazard assessment in the basin of Athens Metropolitan city, Greece. Environmental Earth Sciences, v. 75, p. 319, 2016.

BORGES, M.P., CRUVINEL, A.S., FLORES, W.M.F., BARBOSA, G.R. Utilization of geoprocessing techniques for the elaboration of flood level: a case study of the ecological park of the Paranaíba river. In: XVII SIMPÓSIO BRASILEIRO DE SENSORIAMENTO REMOTO, 2015, Paraíba. Anais. p. 5897-5903. Disponível em: http://www.dsr.inpe.br/sbsr2015/files/p1215.pdf. Acessado em 02 abr 2019.

BRAGA, S.E., WANDERLEY, R.A., HOLANDA, T.F., CALDAS, A.M. Aplicação da Análise Hierárquica para mapeamento de risco de inundação: 0 caso no município de Limoeiro (Pernambuco - Brasil). Meio Ambiente (Brasil), v.2, p. 057-066, 2020 .

Camargo Júnior, A.A., Camargo, D.M., Silva, M.C., Marques, M.L. Vulnerabilidade socioambiental na Bacia do Ribeirão Anhumas - Campinas, SP. In: V JORNADA DE GESTÃo E ANÁLISE AMBIENTAL DA UFSCAR, 2018, São Carlos, SP. Anais. p. 131-140. Disponível em: https://www.researchgate.net/profile/Viviana_Soto_Barrera/publication/346408679_Anais_jornadaaga2018/links /5fc063af9285lc933f64dl43/Anais-jornadaaga2018.pdf\#page=131 Acessado em 05 jan 2020.

CAMPIOLI, P.F., VIEIRA, C.V. Avaliação do Risco a Inundação na Bacia Hidrográfica do Rio Cubatão do Norte, Joinville/SC. Revista Brasileira de Geografia Física, v.12, p. 124-138, 2019.

CAPRARIO, J., RECH, A.S., TASCA, F.A., FINOTTI, A.R. Influence of drainage network and compensatory techniques on urban flooding susceptibility. Water Science and Technology, v.113, p. 1-12, 2019.

CHANG, H.S., CHEN, T.L. Spatial heterogeneity of local flood vulnerability indicators within flood-prone áreas in Taiwan. Environmental Earth Sciences, v. 75, p. 1484, 2016.

COELH0, A. L. N. Método de análise hierárquica (AHP) aplicado a inundação urbana após evento de chuva concentrado. GeoFocus (Artículos), v. 20, p. 183-199, 2017.

DAHRI, N., ABIDA, H. Monte Carlo simulation-aided analytical hierarchy process (AHP) for flood susceptibility mapping in Gabes Basin (southeastern Tunisia). Environmental Earth Sciences, v. 76, p. 302, 2017.

DALFI, R.L., SANTOS, A.R., MOREIRA, T.R., MAGALHÃES, I.A.L., OLIVEIRA, F.S., PEREIRA, M.L. Different methodological scenarios for the mapping of flood areas in the districts of Alegre, ES. In: XVI SIMPÓSIO BRASILEIRO DE SENSORIAMENTO REMOTO, 2013, Paraná. Anais. p. 6885-6892. Disponível em: http://marte2.sid.inpe.br/col/dpi.inpe.br/marte2/2013/05.28.23.21.43/doc/p0370.pdf. Acessado em 05 mai 2019. 
DEAL, B., PALLATHUCHERIL, V. Sustainability and Urban Dynamics: Assessing Future Impacts on Ecosystem Services. Sustainability, v. 1, n. 3, p. 346-362, 2009.

FAISAL, I.M., KABIR, M.R., NISHAT, A. Non-structural flood mitigation measures for Dhaka City. Urban Water, v. 1, p. 145-153, 1999.

FARIA, D.G.M., AUGUSTO FILHO, 0. Aplicação do processo de análise hierárquica (AHP) no mapeamento de perigo de escorregamentos em áreas urbanas. Revista do Instituto Geológico, v. 34, n. 1, p. 23-44, 2013.

IBGE. Instituto Brasileiro de Geografia e Estatística. Conheça as cidades e estados do Brasil. 2020. Avaliado em: https://www.ibge.gov.br/cidades-e-estados/rj/duque-de-caxias.html. Acessado em 15 set 2020.

IPCC. Intergovernmental Panel on Climate Chance. Managing the Risks of Extreme Events and Disasters to Advance Climate Chance Adaptation (SREX). 2012. Avaliado em: https://www.ipcc.ch/report/managing-the-risks-ofextreme-events-and-disasters-to-advance-climate-change-adaptation/. Acessado em 20 out 2019.

IORIS, A.A.R., COSTA, M.A.M. The Challenge to Revert Unsustainable Trends: Uneven Development and Water Degradation in the Rio de Janeiro Metropolitan Area. Sustainability, v. 1, n. 2, p. 133-160, 2009.

FEMA. Federal Emergency Management Agency. Guidelines and Specifications for Flood Hazard Mapping Partners. 2003.

KAUR, H., GUPTA, S., PARKASH, S., THAPA, R., MANDAL, R. Geospatial modelling of flood susceptibility pattern in a subtropical área of West Bengal, India. Environmental Earth Sciences, v. 76, p. 339, 2017.

KLAUSS, S., KREIBICH, H., MERZ, B., KUHLMANN, B., SCHROTER, K. Large-scale, seasonal flood risk analysis for agricultural crops in Germany. Environmental Earth Sciences, v. 75, p. 1289, 2016.

KOBIYAMA, M., et al. Prevention of natural disasters: basic concepts. Curitiba, Organic Trading. 109p. 2006.

LEAL, F.C.B.S., BARBOSA, I.M.B.R., AQUINO, J.T. Mapeamento de áreas vulneráveis à inundação com uso do SIG e da análise multicritério: o caso da Bacia Hidrográfica do rio Una em Pernambuco. Revista Gestão \& Sustentabilidade Ambiental, v. 9, p. 20-40, 2020.

LUZ BARCELLOS, P.C., et al. Meteorological diagnosis of the natural disasters that occurred in the last 20 years in the city of Duque de Caxias. Revista Brasileira de Meteorologia, v. 31, n. 3, p. 319-329, 2016.

LUZ BARCELLOS, P.C., COSTA, M.S., CATALDI, M., SOARES, C.A.P. Management of non-structural measures in the prevention of flash floods: a case study in the city of Duque de Caxias, state of Rio de Janeiro, Brazil. Natural Hazards, n. 89, p. 313-330, 2017.

MAGALHÃES, I.A.L., THIAG0, C.R.L., AGRIZZI, D.V., SANTOS, A.R. Use of geotechnologies for mapping flood risk areas in Guaçuí, ES: a comparative analysis between two methods. Geoscience Notebooks, v. 8, n. 2, p. 63-70, 2011.

MARCELINO, E.V. Natural disasters and geotechnologies: basic concepts. Rio Grande do Sul, Teaching Notebook ${ }^{\circ} 1$. 2007.

MARENG0, J.A., JONES, R., ALVES, L., VALVERDE, M. Future change of temperature and precipitation extremes in South America as derived from the PRECIS regional climate modeling system. Internacional Journal of Climatology, v. 29, p. 2241-2255, 2009.

MIRANDA, N.M.G., CAPRARIO, J., MARTINI, L.C.P., FINOTTI, A.R. Processo Hierárquico Analítico (AHP) em Modelagem Espacial da Vulnerabilidade à Inundação em Ambientes Mistos. Anuário do Instituto de Geociências- UFRJ, v. 42, n. 4, p. 172-181, 2019.

MIRZAEI, G., SOLTANI, A., SOLTANI, M., DARABI, M. An integrated data-mining and multi-criteria decision-making approach for hazard-based object ranking with a focus on landslides and floods. Environmental Earth Sciences, v. 77 , p. 581, 2018. 
MONTEZUMA, R.C.M., OLIVIERA, R.R. Nature and society in the municipality of Duque de Caxias: the meanings of the empty spaces. Educação Ambiental - Duque de Caxias, NIMA/PUC-Rio, Rio de Janeiro, p. 93-110, 2009.

NCAR. National Center for Atmospheric Research. Flash Flood Early Warning System Reference Guide. University Corporation for Atmospheric Research - NCAR. 2010. Disponível em: https://www.meted.ucar.edu/sign_in.php?go_back_to=/communities/hazwarnsys/ffewsrg/FF_EWS.pdf. Acessado em 03 nov 2019.

NOGUEIRA, T.P.N. Mapeamento da suscetibilidade à inundação na bacia hidrográfica do ribeirão da Fábrica, município de Patos de Minas MG. Uberlândia, 2017. 123f. Dissertação (Mestrado em Qualidade Ambiental), Universidade Federal de Uberlândia. Minas Gerais. 2017.

PREVOT, R., MARTINS, C.M.F.L., BARROS, D.F., COSTA, A.S.M. The Historical Trajectory of Duque de Caxias and the Constitution of Social Space from the Advent of REDUC: Paradoxes and Contradictions. In: REUNIÃ0 DE ESTUDOS ORGANIZACIONAIS, 2014, Rio Grande do Sul. Avaliado em: http://www.anpad.org.br/admin/pdf/2014_EnE0365.pdf . Acessado em 05 mai 2019.

RODRIGUES, A.C., GUNTHER, W.M.R., VASCONCELLOS, M.P., GIULIO, G.M., BOSCOV, M.E.G. Delineamento da produção científica sobre desastres no Brasil no início deste século. Desenvolvimento e Meio Ambiente, v. 34, p. 61-73, 2015.

SAATY, T.L.A. Scaling method for priorities in hierarchical structures. Journal of mathematical psychology, v. 15, p. 234-281, 1977.

SAATY, T.L. The Analytic Hierarchy Process. McGraw Hill, New York. 1980.

SAATY, T.L. How to make a decision: The analytic hierarchy process. European Journal of Operational Research, v. 48, p. 9-26, 1990 .

SAATY, T.L. Decision making with the analytic hierarchy process. International Journal of Services Sciences, v. 1, n. 1, p. 83-98, 2008.

SANTOS, A.R., LOUZADA, F.L.R., EYGÊNIO, F.C. ARCGIS 9.3 total: applications for spatial data. Espírito Santo: CAUFES. 2010.

SHAHABI, H., HASHIM, M., AHMAD, B.B. 2015. Remote sensing and GIS-based landslide susceptibility mapping using frequency ratio, logistic regression, and fuzzy logic methods at the central Zab basin, Iran. Environmental Earth Sciences, 73: 8647-8668.

SILVEIRA, R., SANTOS, S.O., ROSSI, A.P., EUGENIO, F.C., SANTOS, A.R. Mapping áreas of flooding in Horizonte stream sub-basin, municipality of Alegre, ES. Forest Institute Journal, v. 25, n. 2, p. 179-191, 2013.

SUN, D., ZHANG, D., CHENG, X. Framework of National Non-Structural Measures for Flash Flood Disaster Prevention in China. Water, v. 4, n. 1, p. 272-282, 2012.

TEBALDI, C., HAOHOW, K., ARBLASTER, J., MEEHL, G. Going to extremes. An intercomparison of model-simulated historical and future changes in extreme events. Climatic Change, v. 79, p. 21-185, 2007.

TOMINAGA, L.K., SANT0R0, J., AMARAL, R. Natural disasters: know to prevent. São Paulo: Geological Institute. 2009.

WM0. World Meteorological Organization. Final report on flood hazard mapping project. 2009. Avaliado em: http://typhooncommittee.org/docs/publications/WM0 TD1519_2009.pdf. Acessado em 20 out 2019.

YOUSSEF, A.M., PRADHAN, B., SEFRY, S.A. Flash flood susceptibility assessment in Jeddah city (Kingdom of Saudi Arabia) using bivariate and multivariate statistical models. Environmental Earth Sciences, v. 75, p. 12, 2016.

ZÊZERE, J.L., PEREIRA, S., TAVARES, A.0., et al. Disaster: a GIS database on hydro-geomorphologic disasters in Portugal. Natural Hazards, v. 72, p. 503-532, 2014. 